\title{
(2) OPEN ACCESS \\ Leadership reflections a year on from the rapid roll- out of virtual clinics due to COVID-19: a commentary
}

\author{
Anthony W Gilbert (D) , 1,2 Lucy Davies, ${ }^{3}$ John Doyle, ${ }^{1}$ Saroj Patel, ${ }^{4}$ Luke Martin, ${ }^{3}$ \\ Deepak Jagpal, ${ }^{5}$ Joe C T Billany, ${ }^{3}$ John Bateson (id ${ }^{6}$
}

${ }^{1}$ Therapies Department, Royal National Orthopaedic Hospital Stanmore, Stanmore, UK

${ }^{2} S$ chool of Health Sciences, University of Southampton,

Southampton, UK

${ }^{3}$ Operations Team, Royal National Orthopaedic Hospital Stanmore, Stanmore, UK ${ }^{4}$ Digital Team, Royal National Orthopaedic Hospital Stanmore, Stanmore, UK

${ }^{5}$ Information Governance Team, Royal National Orthopaedic Hospital Stanmore, Stanmore, UK

${ }^{6}$ Improvement Team, Royal National Orthopaedic Hospital Stanmore, Stanmore, UK

\section{Correspondence to} Anthony W Gilbert, Therapies Department, Royal National Orthopaedic Hospital Stanmore, Stanmore HA7 4LP, UK; anthony.gilbert@nhs.net

Received 13 August 2020 Revised 22 March 2021 Accepted 7 May 2021 Published Online First 26 May 2021

\begin{abstract}
COVID-19 changed the way we delivered care to our patients at our Hospital. Prior to the pandemic, no patient facing video clinics and only a small number of telephone clinics were held. In this paper, we share our experience of rapidly implementing virtual clinics (VCs) due to COVID-19. This commentary is based on focused discussions between hospital leaders and provides a reflective account and commentary on leadership lessons learnt from our experience of deploying VCs. We outline success factors (being able to capitalise on existing strategy, having time and space to establish VCs, using an agreed improvement framework, empowering a diverse and expert implementation team with a flat hierarchy, using efficient decision pathways, communication and staff willingness to change), technical challenges (patient capability and skills to use technology, patient connectivity and platform capacity) and considerations for the future (sustaining new ways of working, platform selection, integration, business continuity and commissioning considerations, barriers regarding capability and communication, effectiveness and clinical outcomes). Finally, we provide an overview of the leadership lessons from this project and identify key areas of focus for delivering successful change projects in future (the vision, allocation of resources, methodology selection and managing the skills gap).
\end{abstract}

\section{INTRODUCTION}

COVID-19 changed the way we delivered care to our patients at the Royal National Orthopaedic Hospital (RNOH). Prior to the pandemic, only a very small proportion of telephone clinics were held with no video clinics. On 5 March 2020, the organisation set a target to deliver $80 \%$ of clinics virtually in response to the COVID-19 pandemic, to be achieved by 16 March 2020 . The results are published elsewhere ${ }^{1}$; in summary, the goal was achieved within 3 weeks through a rapidly deployed process of improvement using the Institute for Healthcare Improvement (IHI) approach. ${ }^{2}$ In this paper, we have attempted to share our experience of rapidly implementing virtual clinics (VCs) to 'lock in' our learning. ${ }^{3}$ This commentary paper is based on focused discussions between $\mathrm{RNOH}$ leaders where we attempt to highlight key considerations to enable future success in similar programmes.

\section{SUCCESS FACTORS}

Increasing $\mathrm{VCs}$ at the $\mathrm{RNOH}$ was an existing part of the 2019/2020 Operational Strategic Plan in line with the National Health Service (NHS) Long Term Plan. ${ }^{4}$ In November 2019, 4 months before the COVID-19 response began, the operational management team agreed a platform for video clinics. This was launched on 27 February 2020. Senior leaders at RNOH committed to increase VC activity in advance of the project, meaning the project was organisationally sanctioned and supported, with goals clearly communicated throughout the Trust. These preparations for VC provided helpful groundwork for the rapid implementation process. The RNOH does not have an emergency department so was not subject to an immediate surge of patients with COVID-19, although the Trust did establish a new emergency orthopaedic trauma referral pathway enabling acute trusts to free up bed capacity elsewhere in London. ${ }^{5}$ There was also a strict limit on the type of activity that could take place at the hospital to help to minimise the spread of COVID-19. This led to an overall decrease in activity through the hospital. In this context, the VC implementation team were afforded a short period of protected time to implement VC.

The $\mathrm{RNOH}$ previously committed to applying the $\mathrm{IHI}^{2}$ approach to quality improvement (QI) to all applicable change processes and established an improvement team to support it's delivery. The IHI method provided a clear process to interrogate the change being proposed and for thinking through, conducting and analysing the change ideas in a Plan-Do-Study-Act cycle. The decision to invest in the use of high-quality improvement science approaches across the organisation has been made to achieve our organisational goal: 'To be a world-leading orthopaedic hospital with the best patient care and staff experience in the $\mathrm{NHS}^{\prime 6}(\mathrm{p} 18)$. Improvement science is at the centre of enabling us to achieve this goal.

The VC implementation team had members from clinical practice, research, improvement, operational management, project management and volunteer services and were sanctioned by the organisation to lead these changes. One of the project team is actively researching this area. ${ }^{7-9}$ The multidisciplinary team was able to make decisions and met daily to maintain a fast pace. This partnership working, drawing diverse individuals from across the organisation, pooled a range of knowledge and skills that were essential to the success of this project. Staff across the trust had access to this expertise via floor walkers on the ground, often consisting of core members of the implementation team, and this established clear, responsive and open lines of communication between the implementation team and those delivering the service. 
In the initial phases, two daily meetings were held (virtually or socially distanced face to face) to capture lessons learnt and to agree the next day's actions.

Implementation of new methods of $\mathrm{VC}$ required governance and close collaboration with corporate service stakeholders such as the Information Governance Team (IG) and Digital Services Team. Wherton et al's guidance on introducing virtual consultations emphasises the need to maintain ongoing dialogue with Information Technology (IT) teams. The need to be flexible during the pandemic led to changes in IG guidance as NHSx advised that it is acceptable to use free videoconferencing tools such as Skype, WhatsApp and/or FaceTime. ${ }^{10}$ The project was conducted in collaboration with these internal RNOH stakeholders but processes were not centrally controlled or rigid. The limited bureaucracy, and the additional allocation of resources and the increased flexibility of the organisation to make space for $\mathrm{VC}$ enabled the team to move at pace. This was possible because VC implementation in response to COVID-19 was a common purpose with clear communication between governance functions and the implementation team; decisions were quickly made and devolved whenever possible. Leaders trusted staff to make appropriate decisions and established a flattened hierarchy. The multidisciplinary team, with diverse expertise and knowledge, was trusted to operate efficiently and effectively, drawing in a wide range of views, and embracing constructive criticism. The implementation team was comprised of multiple professionals from a range of backgrounds and was neither limited by a single-centralised team's capacity, nor slowed down by any one decision-maker.

The aim of the project was communicated widely, consistently and clearly. All-staff communications via email and the intranet explicitly stated, on 5 March 2020, that there was a target of $80 \%$ VC by 16 March 2020 and that all nonessential face-to-face (F2F) appointments must cease due to COVID-19. This empowered the implementation team to drive the roll-out. The implementation team worked closely with the clinicians to ensure that insights from front-line staff were captured and acted on. 'Good news' stories were supplemented with accessible data and shared across all staff via email. These stories were celebrated to facilitate engagement elsewhere within the Trust. This is in accord with Woods et al's functional framework for change leaders ${ }^{11}$ who highlight the importance of inspiring others to want to make the change through generating enthusiasm and motivating commitment. Social persuasion has been cited as an important component of self-efficacy theory. ${ }^{12}$ Senior medical backing from the RNOH's Chief Medical Officer regularly highlighted the work frequently within communications to all staff, which helped emphasise the importance and relevance of the work to a wide audience. Establishing an effective learning system allowed the implementation team to learn from these data and present accessible data visualisations, reflecting consistent progress through growing numbers and consolidating performance accomplishments. ${ }^{12}$ Enthusiastic clinicians operating as champions of VC has been shown to be an important success factor in VC implementation, whereas those who are unwilling to try it may inhibit implementation. ${ }^{13}$ In Greenhalgh et al's study of implementation of $\mathrm{VC},{ }^{13}$ clinicians were required to take on a number of new roles and practices such as triaging suitability of patients, finding space for VC, troubleshooting IT and set-up. The mechanisms of bringing about change can be explained by Normalisation Process Theory, ${ }^{14}$ which focuses on the 'work' of implementation. Ongoing, sustained VC implementation will require ongoing, sustained changes to the roles and practices of clinicians. If clinicians are not willing to do the 'work', VC implementation is unlikely to be sustained.

Staff across the Trust demonstrated remarkable flexibility despite the high demands and short notice that the COVID-19 response required. The Trust initially instituted a command and control style of incident management, ${ }^{15}$ establishing a central incident command centre and leadership hierarchy. Alongside this, however, detailed decision-making was often delegated to front-line clinical leaders. Multiple rapid workstreams were established and decisions were quickly made through a series of daily meetings and cascaded to strategically placed staff members to execute agreed actions. Daily learning was shared with the hospital leadership, which enabled them to act on the insights gained from front-line staff. The implementation team acted in a similar role to 'bedside learning coordinators', which have been described elsewhere and have been celebrated as an effective learning system during the COVID-19 pandemic. $^{16}$

The $\mathrm{RNOH}$ has a vision to have the best staff experience in the NHS. ${ }^{17}$ Leadership courses and mentorship programmes have been made available for staff at most levels, and this may have enabled the devolved leadership witnessed during the pandemic. The value of developing and nurturing leadership at all levels of seniority throughout the organisation has been clearly demonstrated.

\section{TECHNOLOGICAL CHALLENGES}

The pandemic pressures that created the conditions for the rapid roll-out also meant that testing phases were shorter than would have been previously been planned. The implementation team concentrated resources and IT support at the beginning of the project timeline to support engagement and help to create a knowledge base among staff. This knowledge base enabled peer learning and troubleshooting, lessening the burden on IT infrastructure in the longer term. This shift in work meant that staff delivering VCs were often required to troubleshoot at the same time. Patients who had difficulty with the technology, or did not know how to use the technology, often required significant support to be able to establish a video call with their clinician. The time spent troubleshooting technical issues was seen as a barrier to telehealth by allied health clinicians during the COVID-19 pandemic ${ }^{18}$ and it also took away from clinical time and would have been better managed by IT or service experts. Strong links between clinical staff and the IT support and implementation teams were required to support clinicians to escalate troubleshooting issues when these interfered with service delivery.

In some cases, the video call was abandoned. Similarly, some patients had poor internet connectivity and low-quality audio and video that led to suboptimal interactions between patient and clinician resulting in an unsatisfactory patient and clinician experience ${ }^{1}$ that is unlikely to have been an effective replacement for an F2F interaction. Poor connectivity can lead to latency, the technology-generated transmission delay, which causes participants to perceive silence at points where talk should occur. ${ }^{19}$ Different styles of communication are required in remote consultations $^{20}$ and with the accelerated implementation of VC, patients and clincians had to adapt their approach. Focus on the different types of talk required (such as social, clinical and operational) are important to support longer-term VC use. ${ }^{21}$ At times, the video consultation platform was not able to cope with the rapid growth nationally and ran out of capacity. These technical challenges led to changes in the patient and clinician interaction 
and impacted on the enthusiasm and commitment of individuals to make the change to VC. ${ }^{11}$

\section{CONSIDERATIONS FOR THE FUTURE}

This period of rapid implementation occurred during a worldwide pandemic event and the sustained high pressure helped maintain the pace of change. The roll-out of VC was one of many initiatives that was being asked of staff and there is a real danger, as staff become more exhausted with the stress of everyday work during the pandemic, ${ }^{22}$ that we are unable to sustain some of these positive changes beyond the immediate response to COVID-19. In conversations about what the "new normal' beyond COVID-19 might look like, we need to ensure that there are positive legacies from this challenging time, ${ }^{23}$ while undertaking the important work of reprioritisation and resource allocation as we look to normality, ${ }^{24}$ as well as addressing the backlog of orthopaedic procedures. The organisation recognises that sustained implementation requires ongoing commitment from staff; the creation of a new 'Wellbeing Lead' intends to provide support to staff to reset beyond the pandemic and create the capacity for agency for future initiatives.

Embedding change can be a major challenge, ${ }^{25}$ particularly as the crisis which created the conditions for the early successes at $\mathrm{RNOH}$ abates. The approach should seek to build on the legacy of flattened hierarchies and front-line leadership ${ }^{26}$ where all staff champion and drive appropriate use of VC. This requires engagement and feedback from clinicians and patients during any evaluation. The implementation team was sanctioned by the organisation to implement changes, and were therefore seen to have cognitive authority, defined by Hunt and May as being seen by others to 'possess qualities of competence, trustworthiness and credibility in meeting their accountabilities' ${ }^{27}$ Cognitive Authority Theory ${ }^{27}$ explains negotiation processes in which individuals manage important relational aspects of inequalities in power and expertise. An understanding of these processes is important for change management in the post COVID-19 era; should rigid hierarchies be introduced and strict performance targets imposed, inequalities of power and the potential for individuals (such as front-line leaders) to mobilise resources for change will be diminished. Heimans and Timms ${ }^{28}$ offer a useful insight into the balance of power; 'old power' is described as working like a currency and held by few, whereas new power is described as operating like a current and made by many. The Large Scale Change model recognises that transformational change is more likely to happen cross-organisationally, and that hierarchical levers can be ineffective as driving change across the wider system. ${ }^{29}$ Within our experience of rapid implementation of VCs, delegating the power and cognitive authority to a range of professionals across the RNOH was key to the success of the project.

Work to redesign non-admitted pathways and consideration of how VC can be used effectively throughout both surgical and non-surgical pathways must be undertaken. The positives associated with VC need to be fully understood and built on. For example, at the RNOH, many patients travel from across the country to use specialist services making $\mathrm{VC}$ a vital tool to overcome geographical boundaries. Additionally, the $\mathrm{RNOH}$ is a largely COVID-secure (green) site, which creates a new incentive to maximise VC. These drivers to change must be communicated to generate enthusiasm, to motivate commitment and inspire others to want to do it, ${ }^{11}$ to move towards a vision that is better and fundamentally different from the prepandemic norm. ${ }^{29}$
We have identified occasions where it would be advantageous to run group sessions, for example, group exercise classes or education classes. While there are many off the shelf applications and many of them lend themselves to group or 1:1 format, there does not appear to be a solution that works for all scenarios. Alternative platforms have been trialled to support the implementation of group sessions within a virtual pain management and rehabilitation programme and we have shared lessons across the organisation to facilitate the spread of quality improvement. It would be beneficial for any VC platform to be integrated with our other hospital systems while being user-friendly and safe. Flexible platforms that can be specifically tailored to suit local needs are needed.

Many VC platforms offered their services for free or under large scale licenses during the pandemic. Work is required to carry out an options appraisal of the platforms available with clinical input to ascertain the medium to long-term strategy. This also needs to take into consideration business continuity planning. Careful contract management will be required during these early stages: organisations need to have contingencies in place should the primary platform fail. NHS leaders and technology providers might consider working together to provide collaborative solutions; a coordinated and potentially centralised effort to procure effective solutions might be the most effective way to address both value and reliability concerns.

Financial appraisal of services is complex. Previous attempts to establish VCs have failed due to non-F2F appointments receiving lower payments. The tariff for VC is currently the same as for F2F, providing equal financial incentive (or disincentive) for either modality. Anecdotally, assumptions are made about VC being 'cheaper' than F2F clinics. The operation of F2F and $\mathrm{VC}$ in an outpatient setting requires similar staffing levels; clinics continue to operate for the same amount of allotted time and there continues to be a requirement for additional support services. As highlighted in our early implementation, patients often require additional levels of support when using VC compared with traditional clinics. If the required resources to implement change is judged greater than the gains, it is unlikely VC will be incorporated as part of routine practice. If, for example, resources diminish through reduction of non-F2F tariffs post COVID-19, hospitals will be financially disincentivised to continue to offer VC. Any amendments to contracts should be made after a thorough and robust economic evaluation to ensure tariffs are appropriate and commensurate with services being offered.

Some patients struggled with getting the technology to work. For example, the platform requires a specific browser and some patients did not understand the difference between browsers. Many patients had to be talked through the process of updating phone or tablet software-again, there were some who did not understand this. $\mathrm{RNOH}$ staff involved in the early implementation were able to contact patients who were missing at the allotted time and provide one-to-one troubleshooting advice. Were this not available, a significant proportion of patients would have missed out on their clinical appointment. Innovations such as a single NHS Patient Helpdesk with opportunity for 'trial runs' would facilitate equity across the NHS. The use of VC changed what was required of patients and this could potentially be burdensome. ${ }^{8}$ Some patients chose phone calls rather than video appointments because they did not have access to the required technology. We need an improved understanding of the barriers to access.

Patients who required support with communication (eg, via an interpreter) may be excessively disadvantaged from forced use 
of VC during COVID-19. VCs represent a good opportunity for translation services to change their ways of working to become more agile (eg, benefit from homeworking). In addition, family members or friends, where appropriate, can be brought into VCs and can help with translation and greater understanding. Clinics with an interpreter present may be limited as patients do not have access to the interpreter to clarify understanding after a consultation is over whereas with family members/friends, patients have continued access to ask questions and allay anxiety. VC may lead to safeguarding issues through not being able to know, or control who was present at a consultation and it is important to ensure safeguarding policies at institutions consider issues arising from the use of VC. ${ }^{30}$

Thorough evaluation of the effectiveness and safety of virtual management of patients is required to guide ongoing implementation moving forwards. Our work on rapid implementation found that, of the patients who underwent a video call, 44\% of these would opt for a video for their next apppointment. ${ }^{1}$ This figure may be influenced by the COVID-19 pandemic. Design of future pathways must incorporate a thorough investigation of patient preferences. Underlying reasons behind why patients do not prefer VC can then be addressed to support better engagement from patients in the future. In addition, a key understanding into the issues and barriers faced by clinicians will influence the optimal use of $\mathrm{VC}$ for clinical care. This is essential as we continue to manage patients beyond COVID-19.

Our vision for the $\mathrm{RNOH}$ is of continuous improvement as the UK's leading specialist orthopaedic hospital, enhancing our international profile for outstanding patient care. As such, our outcomes matter to us. It is essential that we evaluate our clinical outcomes and continue to operate with patient experience at the centre of our evaluation. Robust evaluation is needed to underpin our future pathway development.

\section{SUMMARY OF LEADERSHIP LESSONS}

There have been unprecedented levels of change across the NHS during the pandemic response. Many have questioned how this amount of change was possible given previous challenges in delivering and sustaining transformation. ${ }^{31}$ The leaders at the $\mathrm{RNOH}$ have reflected on learning from this rapid roll-out to identify key areas of focus for delivering successful change projects in future.

Being able to clearly articulate a vision was, on reflection, a key enabler in this project. Against the backdrop of the existing vision to be the world's leading neuromusculoskeletal hospital, the leadership team set a vision of delivering $80 \%$ VCs. This was specific and ambitious. The message was clear, succinct and communicated to all members of staff at the earliest possible time point. Although the implementation team were not involved with setting the virtual roll-out vision, they were able to bring their knowledge and skills to it to provide leadership for the change process.

Identification and allocation of appropriate resources to the project was a critical success factor in the roll-out. Under normal circumstances resource allocation is challenging as there are always competing priorities. This was heightened during the pandemic response with redeployment of staff to different roles across the Trust and the healthcare system. At the RNOH, a small proportion of staff have dedicated time for involvement in research and improvement initiatives as a core job role. It was essential to have staff with the skills and the time to support this project during its implementation. Additional resources were mobilised by reallocating staff members to the project.
Some staff members were flexibly assigned-they contributed and withdrew as was needed. Many of the staff who were not part of the core team contributed to the work of embedding VCs in practice because improvement is part of the daily job. Without these resources, it is unlikely that the data capture, analysis and project learning ${ }^{1}$ would have happened, and likely that the implementation would have been less rapid.

There are different change models and project management tools to guide service improvement; in common with much of the NHS, the RNOH uses the IHI QI framework. Committing to using one framework ensured those involved in the project had a clear approach and a common language for discussing change. The large-scale change model ${ }^{29}$ provides a complementary route into thinking about some of these issues. The $\mathrm{RNOH}$ senior leaders have all received training on quality improvement methodology and a roll-out to all staff commenced in 2019 ensuring enhanced organisational capability to deliver change. Using a formal common change approach helps reassure senior leaders in the organisation that change is managed through testing and reflection, which in turn makes it easier to devolve decisionmaking and facilitate a shift in power and a more distributed leadership, which is essential for large scale change. ${ }^{29}$ The methodology enabled successful initial implementation and facilitated rapid learning across the organisation and beyond.

There was not enough time to undertake a full training needs analysis of staff or patients prior to the implementation of VCs. It was, therefore, decided by the team that technology-aware support staff would be deployed to each clinic across the organisation. Establishing a log of skills was helpful to direct the realtime development of resources. These resources were updated and shared daily if required. Where clinicians were finding VCs challenging, staff were deployed to support them throughout the clinic as required. Staff who required ongoing support were provided with a named contact who worked with them until they achieved confidence and competence.

Patients were provided with less than a week's notice of the conversion from an $\mathrm{F} 2 \mathrm{~F}$ to a virtual consultation. Some patients, who did not feel confident with a video call, opted for a phone call. Some patients who lacked confidence opted for a video call with technical support. An unfamiliar consultation format may not be desirable for all patients. Our previous research found that offering a trial run, where patients are given the opportunity to test the technology, enhances the acceptability and potential uptake of VC. ${ }^{32}$ Providing test clinics would provide patients with the opportunity to learn how to use the technology without interfering with the clinical encounter. While the implementation of VCs was successful, further work is required to understand the impact on the quality of care patients received. Domains of quality such as safety, effectiveness and patient experience ${ }^{33}$ should be considered in future evaluations of virtual consultations. Future service design needs to be undertaken following thorough engagement with patients as we look to redesign pathways for the future.

\section{CONCLUSIONS}

COVID-19 forced a change in the way we delivered care to our patients at the RNOH. VC was rapidly deployed across the Trust to continue delivering care while avoiding transmission of the virus through unnecessary hospital visits. This paper provides a reflective account and commentary on leadership lessons learnt from the experience of deploying VCs.

Having an existing strategy that targeted VC roll-out meant that some enablement works had been completed. This was 
further supported by an agreed improvement framework and an empowered implementation team with permission, time and space. The effective communication and decision-making pathways and flexibility of clinical and non-clinical staff all worked well. Capacity of the chosen platform, patient connectivity and ability to access and use the technology were challenges to successful delivery. In the future, attention needs to be focused on sustaining these new ways of working and routinising appropriate use of virtual consultations. Consideration must also go to selecting a platform that is stable, fulfils the complex requirements of a multitude of clinical areas and is integrated with Trust systems. Business continuity planning must take place to ensure viable alternatives, and the economics of $\mathrm{VC}$ must be fully understood in the context of commissioned services. Provision must consider the barriers that patients face when accessing care, patient preferences and the suitability of technology to achieve high-quality clinical outcomes.

Twitter Anthony W Gilbert @awgilbert11, Lucy Davies@LucyDaviesC00, John Doyle @JPTDoyle, Luke Martin @lukesbighand, Joe C T Billany @JoeBillany and John Bateson @ImprovementRNOH

Acknowledgements The authors wish to acknowledge several groups of people from the Royal National Orthopaedic Hospital: the patients and clinicians who participated in the virtual clinics, the information technology team for their support with the hardware and software, the information governance team for their expertise on data protection and privacy impact, the Research and Innovation Centre for prompt review and registration of the project and the administration staff for contacting patients. The authors are particularly grateful to the COVID-19 Action Group (Ruth Adam, Rebecca Tobin, Shiv Bagdai, Noreen Galvin, lan Farr and Adam Allain) for their support with the overall delivery of the project.

Contributors AWG and JB designed the work. All authors were involved in collection, analysis and interpretation of data. AWG drafted the work. All authors critically revised the draft, gave final approval of the version to be published and are accountable for all aspects of the work.

Funding This commentary was internally funded. AWG, Clinical Doctoral Research Fellow (ICA-CDRF-2017-03-025), is funded by Health Education England and supported by the National Institute for Health Research (NIHR).

Disclaimer This paper presents independent research funded by the National Institute for Health Research (NIHR). The views expressed are those of the authors and not necessarily those of the NHS, the NIHR or the Department of Health and Social Care.

\section{Competing interests None declared.}

Patient consent for publication Not required.

Provenance and peer review Not commissioned; externally peer reviewed.

Open access This is an open access article distributed in accordance with the Creative Commons Attribution 4.0 Unported (CC BY 4.0) license, which permits others to copy, redistribute, remix, transform and build upon this work for any purpose, provided the original work is properly cited, a link to the licence is given, and indication of whether changes were made. See: https://creativecommons.org/ licenses/by/4.0/.

ORCID iDs

Anthony W Gilbert http://orcid.org/0000-0003-2526-8057

John Bateson http://orcid.org/0000-0002-3620-1387

\section{REFERENCES}

1 Gilbert AW, Billany JCT, Adam R, et al. Rapid implementation of virtual clinics due to COVID-19: report and early evaluation of a quality improvement initiative. BMJ Open Qual 2020;9:e000985.

2 Institute for Healthcare Improvement. Quality improvement essentials toolkit online: Institute for healthcare improvement, 2020. Available: http://www.ihi.org/resources/ Pages/Tools/Quality-Improvement-Essentials-Toolkit.aspx [Accessed 4 Aug 2020].

3 Swaithes L, Dziedzic K, Sharp CA, et al. Context, context, context: how has covid-19 changed implementation globally and how can we 'lock in' learning? Rheumatology 2020;59:1804-7.

4 Department of Health. The NHS long term plan. online, 2019.
5 Iliadis AD, Eastwood DM, Bayliss L, et al. Providing a paediatric trauma and orthopaedics service during the peak of the COVID-19 pandemic: the Royal national orthopaedic Hospital experience. Bone Jt Open 2020;1:287-92.

6 RNOH. RNOH: UK's leading specilaist orthopaedic hospital Online, 2020. Available: https://www.rnoh.nhs.uk/application/files/4416/0076/6584/J001361_-_20-149_ RNOH_2019_20_Annual Report_Complete_Digital release.pdf

7 Gilbert AW, Jones J, Stokes M, et al. Protocol for the connect project: a mixed methods study investigating patient preferences for communication technology use in orthopaedic rehabilitation consultations. BMJ Open 2019;9:e035210.

8 Gilbert AW, Jones J, Jaggi A, et al. Use of virtual consultations in an orthopaedic rehabilitation setting: how do changes in the work of being a patient influence patient preferences? A systematic review and qualitative synthesis. BMJ Open 2020;10:e036197.

9 Gilbert AW, Jones J, Stokes M, et al. Factors that influence patient preferences for virtual consultations in an orthopaedic rehabilitation setting: a qualitative study. BMJ Open 2021;11:e041038

10 NHSx. COVID-19 Ig advice online: NHSx, 2020. Available: https://www.nhsx.nhs.uk/ information-governance/guidance/covid-19-ig-advice/ [Accessed 3 Mar 2021].

11 Woods AL, Luciano MM, Aloia TA, et al. Functional framework for change leaders: results of a qualitative study. BMJ Leader 2020;4:207-13.

12 Bandura A. Self-Efficacy: toward a unifying theory of behavioral change. Psychol Rev 1977;84:191-215.

13 Greenhalgh T, Shaw S, Wherton J, et al. Real-world implementation of video outpatient consultations at macro, meso, and micro levels: Mixed-method study. $J$ Med Internet Res 2018;20:e150.

14 May C. Towards a general theory of implementation. Implement Sci 2013;8:18.

15 Owen C, Scott C, Adams R. Leadership in crisis: developing beyond command and control. Aus J Emer Manage 2015:30:15-19.

16 Shand J, Allwood D, Lee N, et al. Systematically capturing and acting on insights from front-line staff: the 'Bedside Learning Coordinator'. BMJ Qual Saf 2021. doi:10.1136/ bmjqs-2020-011966. [Epub ahead of print: 05 Feb 2021].

17 RNOH. Annual report 2018/2019 online, 2019. Available: https://www.rnoh.nhs.uk/ application/files/4415/7243/1009/j000899_-_19-103_rnoh_annual_report_201819_web-release-aut28-nid1352.pdf [Accessed 3 Aug 2020].

18 Malliaras P, Merolli M, Williams CM, et al. 'It's not hands-on therapy, so it's very limited': telehealth use and views among allied health clinicians during the coronavirus pandemic. Musculoskelet Sci Pract 2021;52:102340.

19 Seuren LM, Wherton J, Greenhalgh T, et al. Whose turn is it anyway? Latency and the organization of turn-taking in video-mediated interaction. J Pragmat 2021; 172:63-78

20 Roberts LC, Osborn-Jenkins L. Delivering remote consultations: talking the talk. Musculoskelet Sci Pract 2021;52:102275.

21 Wherton J, Shaw S, Papoutsi $C$, et al. Guidance on the introduction and use of video consultations during COVID-19: important lessons from qualitative research. BMJ Leader 2020:4:120-3.

22 Launer J. Burnout in the age of COVID-19. Postgrad Med J 2020;96:367-8.

23 Nelson B. The positive effects of covid-19. The BMJ 2020;369.

24 Green G, Eastwood D, Hashemi-Nejad A. COVID-19 and resuming elective paediatric orthopaedics in a London major trauma centre and a tertiary referral centre online: BOA, 2020. Available: https://www.boa.ac.uk/resources/covid-19-and-resumingelective-paediatric-orthopaedics-in-a-london-major-trauma-centre-and-a-tertiaryreferral-centre.html [Accessed 3 Mar 2021].

25 Grimshaw JM, Eccles MP, Lavis JN, et al. Knowledge translation of research findings. Implementation Sci 2012:7.

26 Ellis R, Hay-David AGC, Brennan PA. Operating during the COVID-19 pandemic: how to reduce medical error. Br J Oral Maxillofac Surg 2020;58:577-80.

27 Hunt KJ, May CR. Managing expectations: cognitive authority and experienced contro in complex healthcare processes. BMC Health Serv Res 2017:17:1.

28 Heimans J, Timms H. Understanding "New Power". Harvard Business Review 2014:92:48-56.

29 NHS England. Leading large scale change: a guide to leading large scale change through complex health and social care environments. A practical guide online: NHS England, 2018. Available: https://www.england.nhs.uk/wp-content/uploads/2017/09/ practical-guide-large-scale-change-april-2018-smll.pdf [Accessed 8 Mar 2021].

30 Gilbert AW, Booth G, Betts T, et al. A mixed-methods survey to explore issues with virtual consultations for musculoskeletal care during the COVID-19 pandemic. BMC Musculoskelet Disord 2021:22:245

31 Majeed A, Maile EJ, Bindman AB. The primary care response to COVID-19 in England's National health service. J $R$ Soc Med 2020;113:208-10.

32 Gilbert AW, Jaggi A, May CR. What is the acceptability of real time 1:1 videoconferencing between clinicians and patients for a follow-up consultation for multi-directional shoulder instability? Shoulder Elbow 2019:11:53-9.

33 Department of Health. High quality care for all. NHS next stage final review report online, 2008. Available: https://assets.publishing.service.gov.uk/government/uploads/ system/uploads/attachment_data/file/228836/7432.pdf [Accessed 11 Mar 2021] 